\title{
População idosa e letramento em saúde: reflexões acerca do acesso e da não utilização dos serviços de saúde.
}

Elderly population and health literacy: reflections on access and non-use of health services.

\section{Flavio Ignes Tristão}

Universidade Federal do Espírito Santo, Brasil

flaviotristao@hotmail.com

Conflito de interesses: nada a declarar. Financiamento: nada a declarar.

Data de Submissão: 14/06/2021

Data de Aprovação: 29/07/2021

Todo o conteúdo do JIM é licenciado sob Creative Commons, a menos que especificado de outra forma e em conteúdo 


\section{RESUM0:}

Segundo o prospecto da população mundial das Nações Unidas divulgado em 2019, estima-se que a população total do Brasil é de aproximadamente 210 milhões de habitantes. Destes, cerca de 30 milhões correspondem à população acima de 60 anos, representando, portanto, 14\%. Este fato nos permite notar a considerável expansão da população idosa no transcorrer dos últimos 70 anos, visto que na década de 1950, a população acima de 60 anos representava apenas 4,9\% do total de habitantes.

Ao situar esta dinâmica populacional que envolve o aumento da expectativa de vida dos brasileiros e, consequentemente, de sua população idosa, cabe direcionar uma reflexão acerca do acesso e da utilização do sistema de saúde por parte deste estrato populacional.

Sem pretender buscar aprofundar a íntima relação entre a integralidade da atenção e a disponibilidade dos serviços, vale lembrar que a primeira apresenta uma enorme abrangência, passando pela necessidade de compreender e assimilar o conceito ampliado de saúde, o modelo organizacional do sistema de saúde e o desenvolvimento de políticas de saúde que terão influência direta no cotidiano da população.

Este trabalho surge como um recorte da dissertação de mestrado em que foram investigadas as causas do absenteísmo dos usuários aos serviços de saúde, porém para o público geral. Portanto, agora, os resultados da referida dissertação serão analisados a partir dos participantes entrevistados que tinham mais de sessenta anos, o que compreende dez dos vinte e seis usuários que não haviam comparecido às consultas agendadas.

Ao buscar compreender a utilização dos serviços de saúde, bem como sua "não utilização" (o absenteísmo dos usuários aos serviços de saúde) e as causas deste fenômeno, é necessário entender as formas em que se dão as condições de acesso aos serviços e assim realizar uma conexão entre esta nítida contradição entre os recursos oferecidos e sua não utilização.

Alguns fatores que têm interferência direta no acesso aos serviços de saúde de forma adequada e em tempo oportuno são nesta investigação considerados, tais como a disponibilidade dos serviços, os recursos financeiros envolvidos, o grau de informação deste público e sua aceitabilidade acerca dos diferentes eventos que compõem a produção do cuidado.

Os resultados evidenciam uma necessidade de se avaliarem criteriosamente as dimensões do letramento em saúde para se poder intervir com eficácia e dar o respectivo empoderamento e controle às pessoas e comunidades que, tanto em função do baixo nível educacional quanto da idade avançada, podem apresentar menores níveis de letramento em saúde e, consequentemente, estar mais vulneráveis com relação ao seu estado de saúde.

Palavras-chave: Letramento em saúde; idosos; capacitação; acesso. 


\section{ABSTRACT:}

According to the United Nations World Population Prospect released in 2019, it is estimated that the total population of Brazil is approximately 210 million inhabitants. Of these, about 30 million correspond to the population over 60 years, representing, therefore, $14 \%$. This fact allows us to note the considerable expansion of the elderly population over the last 70 years, since in the 1950s, the population over 60 years old represented only $4.9 \%$ of the total population.

By situating this population dynamic that involves the increase in life expectancy of Brazilians and, consequently, of its elderly population, it is necessary to reflect on the access and use of the health system by this population stratum.

Without intending to seek to deepen the intimate relationship between comprehensive care and the availability of services, it is worth remembering that the first has an enormous scope, including the need to understand and assimilate the expanded concept of health, the organizational model of the health system and the development of health policies that will have a direct influence on the daily lives of the population.

This work emerges as an excerpt from the master's dissertation in which the causes of absenteeism from users to health services were investigated, but for the general public. Therefore, now, the results of that dissertation will be analyzed from the interviewed participants who were over sixty years old, which comprises ten of the twenty-six users who had not attended the scheduled appointments.

When seeking to understand the use of health services, as well as their "non-use" (absenteeism of users to health services) and the causes of this phenomenon, it is necessary to understand the ways in which the conditions of access to services occur and so make a connection between this clear contradiction between the resources offered and their non-use.

Some factors that have a direct interference in the access to health services in an adequate and timely manner are considered in this investigation, such as the availability of services, the financial resources involved, the level of information of this public and its acceptability about the different events that make up the production of care.

The results show a need to carefully assess the dimensions of health literacy in order to intervene effectively and give the respective empowerment and control to people and communities that, both due to low educational level and advanced age, may have lower levels health literacy and, consequently, be more vulnerable in relation to their health status.

Keywords: Health literacy; elderly; training; access. 


\section{ENQUADRAMENTO TEÓRICO}

\section{As dimensões do acesso aos serviços de saúde}

Baseados nos estudos de Thiede, Akewengo e Mclntyre (2007), as quatro dimensões propostas por Sanchez e Ciconelli (2012) são utilizadas como parâmetros mensuráveis do acesso aos serviços de saúde, e correspondem a duas dimensões mais tangíveis, (disponibilidade e poder de pagamento) e duas menos tangíveis (informação e aceitabilidade).

Souza e colaboradores (2014) conceituam o acesso aos serviços de saúde como o maior ou menor grau de facilidade ou dificuldade com que os usuários recebem o cuidado de saúde. De forma mais objetiva, o acesso se situa entre a etapa de procura pelos serviços e sua utilização por parte do usuário.

Para que aconteça um cuidado integral, é necessário que o usuário esteja posicionado no sistema de saúde de forma que seja permitido que este transite entre os diferentes níveis de atenção, sem a existência de barreiras que dificultem o acesso ao cuidado necessário em tempo oportuno.

Já numa abordagem mais direta, Sanchez e Ciconelli (2012) definem que a disponibilidade compreende um conjunto de fatores, tais como: localização geográfica (a distância entre os serviços e os usuários e suas opções de transporte), horário de funcionamento (em relação à disponibilidade de cuidado no momento oportuno), sistemas de marcação (compreende o processo simples, porém por vezes burocrático de ter a consulta agendada), transporte (tanto para que a equipe de saúde possa realizar consultas domiciliares quanto o transporte sanitário de pacientes para diferentes níveis de complexidade), e natureza e extensão da oferta de serviços em comparação às necessidades de saúde da população (o quanto a capacidade instalada da estrutura do serviço tem condições de atender à comunidade).

Observado o aspecto de que a disponibilidade tem relação com a organização do processo de trabalho dos serviços de saúde, Tristão (2016) identificou em sua pesquisa que, no serviço de saúde estudado naquela ocasião, o acesso funcionava em uma relação inversa ao que a dimensão da disponibilidade abrange, uma vez que eram os usuários quem deveriam estar disponíveis ao serviço de saúde em função de sua estrutura organizacional e burocrática.

Ao investigar as causas do absenteísmo dos usuários aos serviços de saúde (ou seja, a não utilização dos serviços), foi constatado que os procedimentos pelos quais os usuários deveriam ser submetidos para acessar os serviços de saúde, em especial aqueles da atenção especializada (atenção secundária), eram marcados por um forte racionalismo presente na forma de burocracia, 


\section{https://doi.org/10.29073/jim.v2i2.428}

normatizações e ações disciplinadoras. Ainda assim, ao usuário que não comparecia às consultas cabia o ônus da culpa (Tristão, 2016).

Assim, pela dinâmica relação entre os usuários e o sistema de saúde que nem sempre se dá de forma alinhada em função da (des)informação, quando há alguma falha nesta interação, os usuários são considerados incorretos e fora da normatividade. Portanto, este comportamento desviante deve ser corrigido com mais educação e informação (ofertados em uma única via pelos profissionais de acordo com seu próprio entendimento do que é correto para diferentes ocasiões) para que possa ser gerado, evidentemente, maior controle sobre os usuários (Tristão, 2016).

Sobre o poder de pagamento, esta dimensão engloba desde o grau de financiamento público do cuidado em saúde até sua relação com a capacidade financeira envolvida nos investimentos diretos e indiretos nos quais os usuários estão sujeitos a se submeterem para terem acesso aos serviços de saúde.

Isso implica, portanto, na análise dos gastos diretos e/ou indiretos feitos pelos usuários para terem suas necessidades de saúde atendidas, como honorários de profissionais de saúde, compra de medicamentos ou realização de exames, transporte ou alimentação, bem como a perda de renda ou produtividade devido ao tempo de espera pelo atendimento (Sanchez \& Ciconelli, 2012).

Para Tristão (2016), a existência de barreiras financeiras precisa ser considerada na análise dos fatores geradores de absenteísmo, uma vez que o poder de pagamento consiste na relação entre o custo direto e indireto da utilização dos serviços envolvendo indicadores como fontes de renda e a capacidade de pagamento por parte do usuário. Assim, por exemplo, o fato de o usuário ter de providenciar uma cópia simples de seus documentos para garantir o agendamento de sua consulta pode desencadear uma série de fatores que terão impacto em suas finanças, pois para além da cópia, pode envolver um deslocamento que pode ou não resultar em gastos extras, além de demandar de um tempo no qual este usuário está impedido de desempenhar alguma atividade laboral, deixando assim, de gerar renda para sua subsistência.

A dimensão da aceitabilidade corresponde à percepção dos serviços pelos usuários de forma individual e coletiva, perpassando pelos aspectos culturais e educacionais e envolvendo fatores como sexo, idade, crenças, condições socioeconômicas, além da confiança no sistema de saúde (Sanchez \& Ciconelli, 2012).

Esta dimensão apresenta um ponto central, que é o respeito mútuo entre os pacientes e os profissionais de saúde, e tem relação direta com a dimensão da informação, pois diz respeito à interação entre as diferentes expectativas tanto dos pacientes quanto dos profissionais na busca pela atenção integral de acordo com as necessidades dos usuários e as condições pelas quais os 
serviços são ofertados. Almeida (2019) destaca que é na "base relacional entre o profissional e o paciente que assenta a comunicação interpessoal, coração da qualidade dos cuidados de saúde " (p. 43).

Para que se estabeleça uma interação que gere uma relação de confiança deve haver um alinhamento entre os profissionais e os usuários, pois, caso o usuário não se aproprie ou não aceite a importância de determinadas condutas, há grandes chances de que o fenômeno da não utilização do sistema ocorra (Tristão, 2016).

Por fim, sobre a dimensão da informação, esta se caracteriza pelo grau de assimetria entre 0 conhecimento do paciente e do profissional de saúde. Portanto, envolve aspectos como a escolaridade, o letramento em saúde e as condições que as pessoas possuem para perceber a própria situação de saúde. Além disso, é a partir da informação que o sujeito desenvolve a capacidade de tomar as próprias decisões relacionadas à saúde (Sanchez \& Ciconelli, 2012).

De acordo com estas variáveis, alguns processos aparentemente simples como compreender os fluxos estabelecidos, entender o motivo de ter sido encaminhado para alguma consulta com outro profissional, aderir ao tratamento, compreender as orientações do profissional da saúde e saber se expressar sobre o próprio estado de saúde podem ocorrer de forma precária (ou até mesmo não acorrer), o que fragiliza as chances de um desfecho favorável para a melhora ou manutenção da saúde.

Considerada como uma ferramenta capaz de reduzir as desigualdades e, inclusive, as disparidades em saúde, a informação caracteriza-se como ponto crucial para o processo de empoderamento das pessoas, para que estas possam ter maior grau de autonomia sobre si e sobre as próprias decisões. De forma mais relevante, a expressão atualmente conhecida por Letramento em Saúde (letramento funcional em saúde ou literacia em saúde) vem sendo valorizada e colocada como peça fundamental para as ações de promoção da saúde, e que, portanto, será alvo da abordagem principal deste artigo juntamente com a análise desta dimensão relacionada ao público idoso.

\section{Informação e Letramento Em Saúde}

Mas, afinal, o que este panorama conceitual sobre as dimensões do acesso aos serviços de saúde poderia realçar sobre o tema proposto a discorrer neste artigo?

Mesmo que o acesso aos serviços de saúde passasse a ser direcionado de forma integral à quimera do modelo em que o usuário é colocado no centro da atenção e da organização do 
processo de trabalho, ainda assim haveria outros fatores que comprometeriam fortemente a capacidade de o sistema ser utilizado de forma otimizada, que são as vulnerabilidades que emergem a partir de algumas condições específicas relacionadas às questões socioeconômicas e culturais, ou seja, a vida como ela é.

O letramento em saúde está relacionado à alfabetização, e implica no conhecimento, na motivação e nas competências das pessoas para acessarem, compreenderem, avaliarem e aplicarem as informações de saúde a fim de fazer julgamentos e tomarem decisões na vida cotidiana relacionadas à saúde no sentido de melhorarem a qualidade de vida durante seu curso (Sørensen et al., 2012).

Diante de uma construção que levou décadas até chegar ao que hoje é conhecido como Letramento em Saúde, Sørensen e colaboradores (2012) sintetizaram dezessete referências entre os anos 1998 e 2009, e chegaram à conclusão de que as definições elaboradas por diferentes Organizações, Associações, Institutos e pesquisadores têm em comum o foco em habilidades individuais, observando aquilo que o indivíduo consegue ou não realizar de acordo com 0 conhecimento que apreende durante a vida.

Para demonstrar a relevância destas habilidades, Arriaga (2019) afirma que o desenvolvimento de estratégias na busca pelo aumento nos níveis de Letramento em Saúde é crucial para a melhora da qualidade de vida, uma vez que mesmo as pessoas com maiores níveis educacionais podem apresentar dificuldades para lidar com a própria condição de saúde que pode se tornar mais vulnerável em função do baixo letramento, bem como para compreender e melhor utilizar 0 Sistema de Saúde no qual estão inseridas.

Outro fato que reforça a importância do Letramento em Saúde é a consideração de sua influência entre as dimensões que interferem no acesso aos serviços de saúde, como afirmam Sanchez e Ciconelli (2012), pois a falta de informação pode se constituir numa lacuna entre a oportunidade de utilização e a real necessidade de utilizar os serviços de saúde.

Por fim, para destacar sua relevância no contexto global, vale citar o fato de que na última Conferência Mundial de Promoção da Saúde realizada em Shanghai em 2016, o Letramento em Saúde foi considerado um dos três pilares para o desenvolvimento das ações de promoção da saúde (OMS, 2016).

Para Sørensen (2019), o Letramento em Saúde caracteriza-se como uma competência crucial para a saúde no século XXI, e considera que seja relevante para todos os profissionais envolvidos com a finalidade de melhorar a vida de outras pessoas, comunidades e sociedades. Além disso, 
é uma ferramenta viável e está em evidência, além de poder ser mensurável por meio de instrumentos específicos.

Assim, um ponto relevante a ser considerado é o fato de que aproximadamente $50 \%$ da população brasileira acima de 25 anos possui escolaridade até o ensino fundamental completo, o que equivale a nove anos de ensino formal, sendo que $32 \%$ não completaram esta formação, podendo, portanto, ter interrompido os estudos entre o primeiro e o nono ano (IBGE, 2019).

Ainda com relação ao nível educacional, a porcentagem da população brasileira acima de 25 anos que completou o ensino médio é de $27 \%$, o que equivale a doze anos de ensino formal. Isso posto, identifica-se que aproximadamente $80 \%$ da população brasileira não acessa o ensino superior (IBGE, 2019).

Este fato tem relação direta com o que fora apresentado anteriormente, pois aborda os aspectos educacionais que interferem significativamente nas condições da população em geral que envolvem 0 processo de tomada de decisões que incide sobre suas escolhas e, consequentemente, sobre a própria saúde.

\section{METODOLOGIA}

Trata-se de um resgate da dissertação de mestrado intitulada "Absenteísmo dos usuários aos serviços de saúde no município de Vila Velha no Estado do Espírito Santo" (TRISTÃO, 2016), porém abordando agora um recorte específico que não havia sido feito até o momento, que é o direcionamento da discussão sobre o fenômeno da não utilização dos serviços de saúde com os dados obtidos nesta pesquisa relacionado ao público idoso.

Um dos objetivos da dissertação consistiu em compreender as causas do absenteísmo no contexto do município estudado, especialmente no que se refere à Atenção Especializada, utilizando a entrevista como ferramenta de coleta de dados, que teve como amostra vinte e seis usuários que não haviam comparecido às consultas agendadas para vinte e seis especialidades médicas no ano de 2015 (Tristão, 2016).

Uma vez que a dissertação em questão analisou este fenômeno voltado para o público geral relacionado às quatro dimensões do acesso descritas anteriormente, este artigo busca um direcionamento ampliado desta análise para compreender a forma como as dimensões do acesso interferem na possibilidade dos idosos em utilizar os serviços disponibilizados, mais especificamente a dimensão da informação (que envolve o Letramento em Saúde), uma vez que 
este foi um dos fatores que mais se mostrou capaz de interferir no desfecho de comparecer ou não às consultas agendadas.

\section{RESULTADOS E DISCUSSÃO}

Sobre o total de entrevistados, dois não possuíam escolaridade (8\%), oito tinham o Ensino Fundamental Incompleto (30\%), seis tinham o Ensino Fundamental Completo (23\%), dois com o Ensino Médio Incompleto (8\%), seis com o Ensino Médio Completo (23\%), um com Ensino Superior Completo (4\%) e um não informou a escolaridade (4\%)

Com relação à idade e escolaridade, dentre os vinte e seis usuários entrevistados, dez deles tinham idade superior a 60 anos, o que representa 38,4\%. Destes, dois não tinham escolaridade, quatro possuíam o ensino fundamental incompleto, dois o ensino fundamental completo, e dois relataram ter o ensino médio completo. Ou seja, o nível de escolaridade de $80 \%$ dos entrevistados acima de 60 anos de idade limita-se ao máximo a nove anos de estudo formal.

Estes dados reforçam o panorama geral apresentado sobre a educação da população brasileira acima dos 25 anos, que aponta que aproximadamente $50 \%$ deste estrato populacional apresenta o ensino fundamental completo (IBGE, 2019).

Porém, para além desta comparação e constatação, é necessário apontar também os desdobramentos que este fato acarreta, pois associado à baixa escolaridade (e, provavelmente, baixo nível de Letramento em Saúde) estão diversos contextos que poderão se configurar como possíveis fatores geradores do absenteísmo.

Apesar de não haver um levantamento nacional sobre o Letramento em Saúde no Brasil, alguns estudos têm apontado um nível de letramento considerado insatisfatório, como o estudo de revisão realizado por Vasconcelos, Sampaio e Vergara (2018).

Um dado relevante neste estudo de revisão é a observação da relação direta entre o nível de escolaridade e o nível de Letramento em Saúde em uma das pesquisas consultadas, que apontou que um dos grupos avaliados apresentava ao menos o ensino médio completo, e o nível de letramento classificado como insatisfatório nos indivíduos deste grupo ocorreu em 13,1\% da amostra. Ou seja, a escolaridade ligeiramente superior aos nove anos correspondentes ao ensino fundamental interferiu na menor incidência do nível de Letramento em Saúde precário (Vasconcelos, Sampaio \& Vergara, 2018). 
Sendo assim, como foi possível observar nos resultados das entrevistas com os usuários, percebe-se que este achado vai de encontro ao que estes estudos têm apontado, o que reforça a tese de que quanto menor for o nível educacional da população, menor o nível de Letramento em Saúde e, consequentemente, maiores os riscos de ocorrerem "ruídos" na comunicação entre os profissionais de saúde e seus pacientes, tornando maiores as chances de que a utilização dos recursos disponíveis (seja uma medicação ou um encaminhamento para consulta em outro nível de atenção) seja feita de forma equivocada, incompleta, ou até mesmo não acontecer.

Mesmo retratando países com perfil demográfico e culturas completamente diferentes do Brasil, pesquisas desenvolvidas em países da Europa confirmam a relação entre a maior idade, baixo status social, menor nível de educação, menor renda e a identificação de piores condições de saúde.

É o caso do estudo feito por Bo e colaboradores (2014) envolvendo quase 30 mil pessoas no centro da Dinamarca, onde foi identificado que de 10 a 20\% da população estudada possuía dificuldades em tarefas relacionadas à capacidade de compreender bem as informações sobre saúde, saber o que fazer e capacidade de se envolver ativamente com profissionais de saúde, enquanto que entre os indivíduos idosos, os que não possuíam a etnia dinamarquesa e com baixo nível socioeconômico, as mesmas dificuldades foram identificadas em torno 40\%. Almeida (2018) sublinha a importãncia do papel dos profisisonais de saúde no incremento dessa compreensão referindo que estes são " a parte mais forte e habilitada da relação terapêutica (p. 36).

Outro dado relevante observado nos resultados encontrados na dissertação por meio das entrevistas com os usuários é o fato de que todos os participantes acima de sessenta anos utilizavam o transporte público como principal meio de locomoção e não possuíam plano de saúde (Tristão, 2016).

Reforçando este aspecto, Machado e colaboradores (2019) indicam que, na maioria das vezes, as pessoas que apresentam níveis precários de Letramento em Saúde são os adultos mais velhos, com baixa escolaridade e que geralmente dependem de programas assistenciais do governo.

Porém, até mesmo pessoas com alto nível de escolaridade podem se deparar com sistemas de saúde de tamanha complexidade que se torna difícil de compreender, o que é agravado por condições que tornem as pessoas mais vulneráveis (Machado et al., 2019).

Seguindo o raciocínio de que a idade avançada está associada ao surgimento de doenças crônicas não transmissíveis e que esta condição associada ao baixo nível educacional pode resultar em escolhas e comportamentos de risco que podem agravar o quadro de saúde em função do baixo nível de Letramento em Saúde (que, como fora exposto, está diretamente relacionado a estes 
fatores), cabe destacar que estes fatores podem se configurar em amplificadores de vulnerabilidades, o que aumenta a sobrecarga dos serviços de saúde em função da assistência a condições de saúde consideradas preveníveis e/ou evitáveis.

Ao citar Panagioti e colaboradores (2018), Veiga (2020) reitera o fato de que o nível de Letramento em Saúde tem influência direta na qualidade de vida, principalmente na população idosa.

Em seu estudo sobre o Letramento em Saúde e a capacitação de idosos na prevenção do Diabetes Mellitus tipo 2 em um centro comunitário de Lisboa, em Portugal, Veiga (2020) contextualiza os impactos que esta doença representa nos serviços de saúde daquele país, ressaltando que há um aumento de $23 \%$ da prevalência desta doença na faixa etária que compreende os idosos de 65 a 74 anos.

Sobre este público em questão, Veiga (2020) identificou por meio de instrumento específico que, 61,9\% dos idosos que participaram da pesquisa têm níveis de Letramento em Saúde inadequados, 28,6\% têm nível problemático e apenas 9,5\% apresentam nível suficiente. Afirma ainda que, em concordância com outros estudos semelhantes, à medida que a idade avança, os níveis de Letramento em saúde tendem a ser mais baixos.

Veiga (2020) cita ainda o estudo realizado por Kim (2009), onde o mesmo também encontrou relação entre o baixo nível de Letramento em Saúde e a incidência de hipertensão arterial e limitação nas atividades de vida diárias.

Apesar do fato de que estes estudos apontam para a relação entre a idade, o baixo letramento em saúde e a incidência de doenças crônicas, outro fator identificado na dissertação relacionado ao letramento em saúde foi a falta de compreensão dos usuários com mais de sessenta anos entrevistados com relação à necessidade de ter sido encaminhado para outra especialidade. Como foi apontado anteriormente, $80 \%$ dos usuários acima de 60 anos entrevistados apresentavam, no máximo, o ensino fundamental completo (Tristão, 2016).

Com base neste aspecto, revela-se a disparidade entre o nível de informação dos usuários e dos profissionais, o que pode comprometer a geração do vínculo e das relações de confiança e, consequentemente, o sentido dado pelo próprio usuário sobre a necessidade de comparecer a uma consulta de determinada especialidade médica, sendo um importante fator gerador de absenteísmo deste público aos serviços de saúde (Tristão, 2016).

Ainda nesse sentido, é possível relacionar a baixa escolaridade com as possíveis fragilidades no conhecimento dos usuários sobre a própria condição de saúde, que pode resultar na criação de uma demanda por parte do usuário que não necessariamente deve ser alvo de alguma 
intervenção, procedimento ou medicalização. Ou seja, de maneira contrária ao que foi apontado acima, níveis precários de letramento em saúde podem fazer com que o usuário crie uma demanda inexistente a respeito da aparente necessidade em saúde, fazendo com que o serviço seja utilizado de forma equivocada, o que pode também trazer riscos para a própria saúde (Tristão, 2016).

\section{CONSIDERAÇÕES FINAIS}

Lançar luz a esta análise do letramento em saúde do público idoso relacionado à capacidade de utilizar ou não os serviços de saúde, permitiu identificar que esta relação também se configura como uma das possíveis causas que contribuem para que a busca pelo cuidado integral tenha 0 seu desfecho prejudicado ou até mesmo impedido.

Percebe-se, portanto, que o perfil dos usuários que participaram da pesquisa permite reforçar as conclusões dos estudos que se dedicam a estudar a mesma temática, ou seja, pode haver uma forte relação entre o baixo letramento em saúde associado à baixa escolaridade e também ao avançar da idade.

Se a escolaridade é um fator que pode tornar vulnerável boa parte da população adulta, o cenário que se desenha para o público idoso certamente envolve mais riscos, o que requer uma atenção diferenciada sobre as abordagens em saúde voltadas para este público específico.

Portanto, cabe fortalecer o movimento em favor da Promoção da Saúde que, caracterizada como política de saúde balizadora das ações a serem desenvolvidas na busca da melhoria da qualidade de vida, empoderamento de pessoas e de comunidades e redução das desigualdades em saúde por meio da associação destas ações aos Objetivos do Desenvolvimento Sustentável, conforme destacado na Conferência de Shanghai em 2016.

Talvez, mais do que nunca, por meio do fomento às ações de Promoção da Saúde vinculadas à ferramenta do Letramento em saúde, estejamos caminhando para uma aproximação mais sensível da necessidade de serem desenvolvidas e implementadas políticas de saúde mais próximas da realidade da vida cotidiana das comunidades e das pessoas que a constituem num território vivo e dinâmico. 
https://doi.org/10.29073/jim.v2i2.428

\section{BIBLIOGRAFIA}

Almeida, C. V. (2018). Literacia em saúde: Capacitação dos profissionais de saúde: 0 lado mais forte da balança. In C. Lopes \& C. V. Almeida (Coords.), Literacia em saúde: Modelos, estratégias e intervenção (pp. 33-42). Lisboa: Edições ISPA. http://bibliografia.bnportugal.gov.pt/bnp/bnp.exe/registo?2016487

Almeida, C. V. (2019). Modelo de comunicação em saúde ACP: As competências de comunicação no cerne de uma literacia em saúde transversal, holística e prática. In C. Lopes \& C. V. Almeida (Coords.), Literacia em saúde na prática (pp. 43-52). Lisboa: Edições ISPA [ebook] - https://repositorio.ispa.pt/handle/10400.12/7662

Disponível em: http://loja.ispa.pt/produto/literacia-em-saude-na-pratica e também em:

https://www.researchgate.net/publication/338503211 BOOK LITERACIA EM SAUDE NA PRATICA [accessed Mar 22 2021].

Arriaga, M. T. (2019) Prefácio: Capacitação dos profissionais de saúde para uma melhor literacia em saúde do cidadão. In: LOPES, C.; ALMEIDA, C. V. (Orgs.). Literacia em Saúde na prática. Lisboa, ISPA: 2019.

Bo, A., Friis, K., Osborne, R. H. \& Maindal, H. T. (2014) National Indicators of Health Literacy: ability to understand health information and to engage actively with healthcare. BMC Public Health v. 14, p. 1095.

IBGE. Instituto Brasileiro de Geografia e Estatística. Pesquisa Nacional por Amostra de Domicílios Contínua 2012 - 2019. Disponível em: https://educa.ibge.gov.br/jovens/conheca-obrasil/populacao/18317educacao.htm|\#: :text=No\%20Brasil\%2C\%20a\%20propor\%C3\%A7\%C3\%A30\%20de, $\% 2 C 8 \%$ 25\%2C\%20em\%202019. Acesso em 20 de Janeiro de 2019.

Machado, S. P., Parente, N. A., Cabral, L. A., \& Henriques, E. M. V. (2019) Letramento funcional em saúde no contexto dos agravos crônicos não transmissíveis. In: Passamai, M. P. B., Sampaio, H. A. C., HENRIQUES, E. M. V. (Orgs.) Letramento funcional em saúde: as habilidades do usuário e o Sistema Único de Saúde. Curitiba: CRV, 2019.

OMS. Organização Mundial da Saúde. Carta de Shanghai. IX Conferência Mundial de Promoção da Saúde. Shanghai, 2016.

Sanchez, R. M. \& Ciconelli, R. M. (2012) Conceitos de acesso à saúde. Rev Panam Salud Publica.;31(3):260-8.

Sørensen K, van den Broucke S, Fullam J, Doyle G, Pelikan J \& Slonska Z, (2012). Health literacy and public health: a systematic review and integration of definitions and models. BMC Public Health 25:80. 
Sørensen, K. (2019) Defining health literacy: Exploring differences and commonalities. In: Okan, 0.; Bauer, U.; Levin-Zamir, D.; Pinheiro, P.; Sorensen, K. (2019) International Handbook of Health Literacy: Research practice and policy across the lifespan. Great Britain.

Souza, L. E. P. F., Guimarães, R., Travassos, C. \& Canabrava, C. M. (2014) Infraestrutura tecnológica do SUS: Rede de estabelecimentos, equipamentos, desenvolvimento científicotecnológico e inovação. In: Paim, J. S.; Almeida-Filho, N. (Org.) Saúde Coletiva: teoria e prática. Rio de Janeiro: Medbook, 2014, p. 211-29.

Thiede M, Akewengo P \& Mc'Intyre D. (2007) Exploring the dimensions of access. In: Mc'Intyre D. \& Mooney G. The economics of health equity. Cambrige-ing: Cambrige University Press, 2007. p. 103-47.

Tristão, F. I. (2016) Absenteísmo dos usuários aos serviços de saúde no município de Vila Velha no estado do Espírito Santo. Dissertação de Mestrado, Universidade Federal do Espírito Santo, Vitória, Espírito Santo, Brasil.

United Nations, Department of Economic and Social Affairs, Population Division (2019). World Population Prospects 2019, Volume II: Demographic Profiles (ST/ESA/SER.A/427).

Vasconcelos, C. M. C. S., Sampaio, H. A. C. \& Vergara, C. M. A. C. (2018) Materiais Educativos para prevenção e controle de doenças crônicas: uma avaliação à luz dos pressupostos do letramento em saúde. Curitiba: CRV, 2018.

Vasconcelos, C. M. C. S., Henriques, E. M. V. \& Sampaio, H. A. C. (2019) Diagnóstico do Letramento Funcional em Saúde. In: Passamai, M. P. B., Sampaio, H. A. C. \& Henriques, E. M. V. (Orgs.) Letramento funcional em saúde: as habilidades do usuário e o Sistema Único de Saúde. Curitiba: CRV, 2019.

Veiga, A. (2020) Literacia em saúde e capacitação do idoso na prevenção da diabetes mellitus tipo2 em contexto comunitário. JIM, v. 1, n. 2, p. 5-17. 